\title{
ANÁLISE DA RESISTÊNCIA DE BLOCOS DE CERÂMICA VERMELHA SOB CONDIÇÕES EXPERIMENTAIS *
}

\author{
G. R. de Oliveira ${ }^{1}$ \\ J. J. N. Silva ${ }^{2}$ \\ O. D. P. Fernandes 3 \\ Foluke Salgado de Assis ${ }^{4}$
}

\begin{abstract}
Resumo
O presente trabalho tem como objetivo analisar as propriedades em blocos de cerâmica vermelha. O diferencial e originalidade em relação ao que já existe em termos científicos são as evidencias de que gradientes de umidade são mais significativos em secagem rápida o que provoca a presença de tensões residuais, como também em termos da porosidade em temperaturas de queima diferentes e conformação sob condições de presença e ausência de vácuo na produção dos blocos. Nestes casos, danos estruturais como trincas, deformações e fraturas podem surgir no produto final ou durante o carregamento de serviço devido à diminuição dos valores de resistência mecânica. Os resultados evidenciam que corpos de prova submetidos à secagem rápida em comparação a secagem lenta, bem como a diminuição da temperatura de queima e ausência de vácuo durante o processo produtivo, decrescem a propriedade mecânica citada..
\end{abstract}

Palavras-chave: Resistência mecânica; Tempo de secagem; Temperatura de queima.

\section{ANALYSIS OF RESISTANCE OF CERAMIC RED BLOCKS UNDER EXPERIMENTAL CONDITIONS}

\begin{abstract}
This study aims to analyze the properties in blocks of red ceramic. The differential and originality in relation to what is already in scientific terms are the evidence that moisture gradients are more significant in the rapid drying causes the presence of residual stresses, but also in terms of porosity in different firing temperatures and conformation under conditions of presence and absence of vacuum in the production of the blocks. In these cases, structural damage such as cracks, deformations and fractures may occur in the final product or service during charging due to the decrease of the mechanical strength. The results show that specimens subjected to rapid drying in comparison to slow drying, and decreasing firing temperature and absence of vacuum during the production process, the mechanical property decrease quoted.
\end{abstract}

Keywords: Mechanical strength; Drying time, Firing temperature.

1 Engenheiro de Materiais, Universidade Federal de Campina Grande, Campina Grande, Paraíba Brasil.

2 Engenheiro de Materiais, Universidade Federal de Campina Grande, Campina Grande, Paraíba Brasil.

3 Engenheiro de Petróleo, Universidade Federal de Campina Grande, Campina Grande, Paraíba Brasil.

4 Engenheiro Metalúrgico, Doutorando em Ciências dos Materiais, Instituto Militar de Engenharia, Rio de Janeiro, Rio de Janeiro - Brasil. 


\section{INTRODUÇÃO}

A extrusão é um processo de conformação industrial que tem se revelado essencial nas indústrias cerâmicas de barro vermelho (telhas e blocos), por se tratar de uma técnica de produção associada a uma elevada produtividade, principalmente para produtos de seção transversal constante (bloco) e muito importante em termos de homogeneização e retirada do ar da massa $(1,2)$. A máquina utilizada para conformação por extrusão é uma extrusora de vácuo também conhecida como "maromba", que é responsável pelo transporte, compactação e extrusão da massa cerâmica; durante o processo o fluxo deve ser o mais constante possível para garantir a qualidade dos produtos (3).

Os blocos cerâmicos, popularmente conhecidos como tijolos, são um dos componentes básicos para a construção civil. Os tijolos são produzidos a partir da argila, geralmente sob a forma de paralelepípedo, possuem coloração avermelhada, apresentam canais/furos ao longo de seu comprimento além de ranhuras e saliências nas faces para facilitara aderência com a argamassa. Há dois tipos de blocos cerâmicos - estrutural e de vedação (2).

Quanto ao preparo da matéria prima, tem-se que todas as operações antes da extrusão podem ser realizadas por processos mecânicos, que compreendem normalmente: trituração, peneiramento, misturadores, amassadores e laminadores. A maior parte dos defeitos dos blocos cerâmicos é em razão desta fase, que influencia todas as outras fases do processo, muitas vezes confundindo o fabricante que não identifica a sua causa (4).

Nas extrusoras dotadas de câmara de vácuo, o ar é retirado da massa, melhorando sua plasticidade com menor consumo de água, facilitando a secagem e aumentando a resistência mecânica do produto verde. A qualidade da extrusão influencia diretamente as propriedades finais do produto verde ou queimado (2).

Em geral, considera-se a resistência dos blocos cerâmicos como um indicador de qualidade e esta depende das matérias primas utilizadas na fabricação, do tempo de secagem e da temperatura de queima; podendo a mesma ser verificada a partir da execução de ensaios mecânicos.

O Objetivo do presente trabalho é analisar as propriedades mecânicas em blocos de cerâmicas vermelhas, conformados por extrusão, em função do uso da câmara de vácuo, do tempo de secagem e da temperatura de queima. 


\section{MATERIAIS E MÉTODOS}

Para o processo de conformação dos blocos cerâmicos foi utilizada uma massa plástica já beneficiada oriunda do município de João Pessoa - Paraíba. Todo processo de conformação foi realizado no Laboratório de Tecnologia de Materiais UAEMa/UFCG.

Inicialmente a massa cerâmica foi laminada, para destruir os aglomerados, conferindo maior homogeneidade a mesma. Em seguida, a conformação dos blocos foi realizada, a princípio com o uso de vácuo e depois sem a utilização do mesmo, utilizando uma extrusora da marca Verdés, conforme mostra a Figura 1:
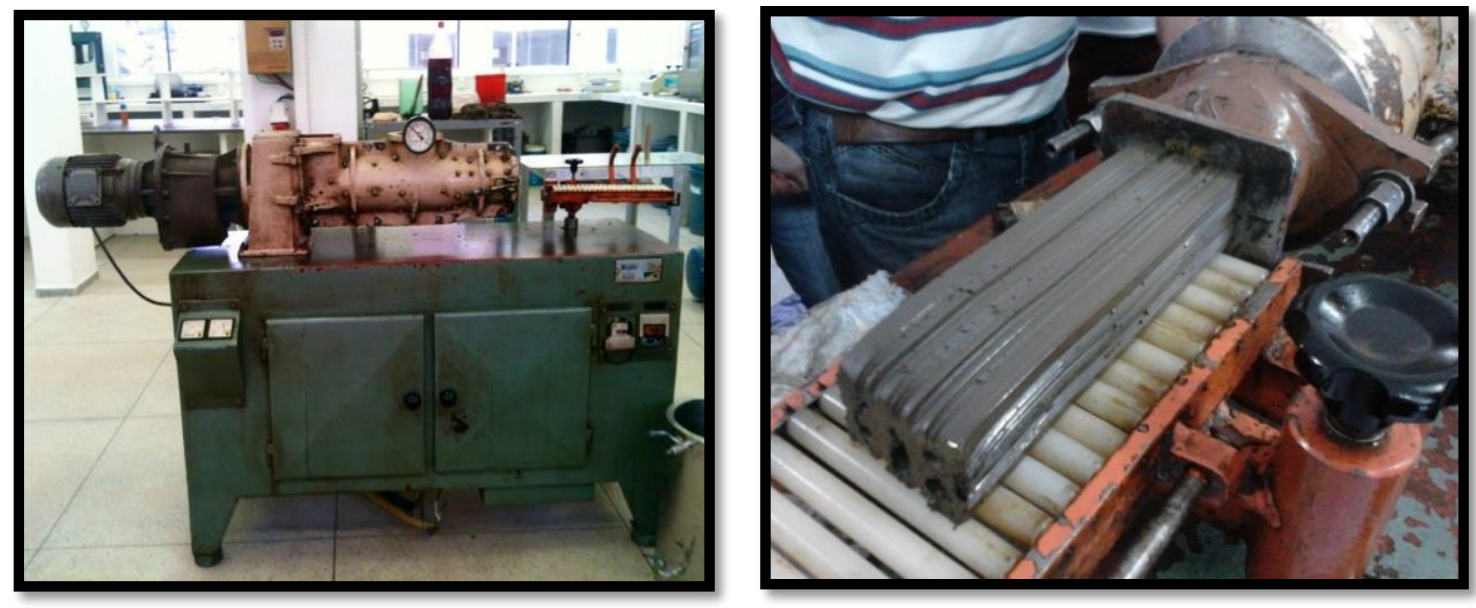

Figura 1 - (a) Extrusora usada para o processo de conformação e (b) conformação dos blocos cerâmicos.

Depois de conformados, os blocos foram então separados em dois grupos de acordo com suas condições de conformação (com vácuo e sem vácuo), e submetidos à etapa de secagem, realizada ao ar livre nas bancadas do mesmo laboratório em que foram conformados.

A secagem é uma fase complexa, pois nela deve-se eliminar a água necessária para a obtenção da massa plástica (5). Quando seco, o bloco está em condições de resistir a transformações físicas e químicas que ocorrem na queima sem danos (6). Para comparação da influencia do tempo de secagem, dois blocos (pertencente ao grupo de conformação com vácuo) foram submetidos à secagem com duração de 24 horas (secagem rápida) e os demais submetidos à secagem com duração de 48 horas (secagem lenta). Após secarem naturalmente, os blocos foram levados à estufa por 72 horas, para serem totalmente secos.

Os corpos submetidos à secagem rápida foram queimados, no forno EDG-3P-S até uma temperatura de $800^{\circ} \mathrm{C}$. Os submetidos à secagem lenta foram separados em dois grupos no qual um grupo foi queimado a $800^{\circ} \mathrm{C}$ e o outro a $1000^{\circ} \mathrm{C}$, ambos contendo blocos conformados com e sem vácuo. O tempo de queima para todos os corpos foi de 1 hora sob uma taxa de aquecimento de $1^{\circ} \mathrm{C} / \mathrm{min}$.

Quanto à queima, esta constitui a operação mais importante na fabricação dos materiais cerâmicos. A sua finalidade é aglomerar as partículas formando uma massa coesa, que traz alterações bastante significantes ao bloco cerâmico, tais como, redução na área específica total e no volume aparente total e aumento da resistência mecânica (7).

Com os blocos consolidados, foram realizados os testes para a determinação da absorção de água, porosidade aparente, massa específica aparente, retração linear e resistência à compressão. 
Para o cálculo da retração linear, separou-se o grupo de blocos queimado a $1000^{\circ} \mathrm{C}$ e mediram-se suas dimensões após a etapa de secagem e após a queima.

\section{Absorção de água e Porosidade Aparente}

Os blocos cerâmicos produzidos foram submetidos à análise de absorção de água, conforme método de ensaio da norma NBR 15270-3/2005 (8).

Para o cálculo de absorção de água e porosidade aparente, todos os blocos tiveram suas massas verificadas (peso após queima). Em seguida, os mesmos foram imersos em água durante $24 \mathrm{~h}$ e novamente pesados (peso saturado). Os blocos também foram pesados imersos na água (Figura 2) segundo o principio de Arquimedes, para o cálculo da porosidade aparente.

Determinada a massa saturada, a massa seca e o peso imerso dos blocos, tiveramse todas as variáveis para achar o índice de absorção de água e a porosidade aparente de cada bloco determinados, respectivamente pelas fórmulas $(A)$ e $(B)$.

$$
\begin{aligned}
& A A(\%)=\frac{\text { Psat }-P Q}{P Q} \times 100 \\
& P A(\%)=\frac{\text { Psat }-P Q}{\text { Psat }-P i} \times 100
\end{aligned}
$$

Onde:

AA é o índice de absorção d'água (\%); PA é a porosidade aparente; Psat é a massa úmida de cada corpo de prova (g); $\mathrm{PQ}$ é a massa seca (após a queima) de cada corpo de prova (g) e Pi é a massa do corpo de prova imerso.

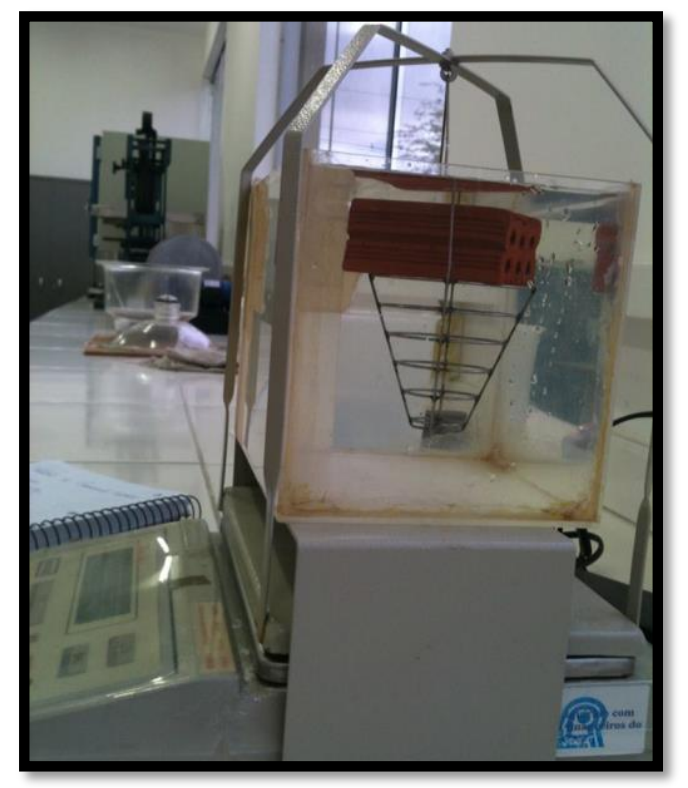

Figura 2. Medição do peso imerso dos blocos cerâmicos.

\section{Massa Específica Aparente}

Para o Cálculo da massa especifica aparente foi utilizada a fórmula (C). 


$$
\operatorname{MEA}(\%)=\frac{P A}{A A} \times 100
$$

Onde:

MEA (\%) é a massa específica aparente; AA é o índice de absorção d'água; PA é a porosidade aparente;

\section{Resistência mecânica}

As propriedades físico-mecânicas dos blocos cerâmicos produzidos foram testadas através da análise de resistência mecânica, conforme método de ensaio determinado pela norma NBR 15270-3/2005.

O ensaio resistência à compressão foi realizado no Laboratório de caracterização de Engenharia de Materiais - UAEMa/UFCG, utilizando uma máquina universal de ensaios modelo DL -10.000 , da marca EMIC. A velocidade de carga utilizada foi de $0,05 \mathrm{MPa} / \mathrm{s}$ e $5 \mathrm{MPa} / \mathrm{s}$.

Antes da realização do o ensaio de resistência à compressão, os blocos primeiramente foram capeados com uma argamassa na proporção de 1:1 (areia e cimento) fazendo com que o bloco obtivesse uma espessura de argamassa em torno de $3 \mathrm{~mm}$, conforme determinado na norma. Depois de a argamassa adquirir uma boa resistência o bloco foi colocado na água, onde permaneceu por 24 horas para então ser rompido. 


\section{RESULTADOS E DISCUSSÃO}

As Tabelas 1-3 mostram os valores referentes às dimensões das faces (largura, altura e comprimento), do peso após a queima (PQ), do peso saturado (Psat) e do peso imerso ( $\mathrm{Pi})$ dos corpos de prova (blocos cerâmicos de vedação).

Tabela 1: Dimensões, peso após queima, peso saturado e peso imerso dos blocos conformados sem vácuo, com vácuo e queimados a $800^{\circ} \mathrm{C}$.

\begin{tabular}{ccccccc}
\hline $\begin{array}{c}\text { Corpo } \\
\text { de } \\
\text { prova }\end{array}$ & $\begin{array}{c}\text { Comprimento } \\
(\mathbf{c m})\end{array}$ & $\begin{array}{c}\text { Largura } \\
(\mathbf{c m})\end{array}$ & $\begin{array}{c}\text { Altura } \\
\mathbf{( c m})\end{array}$ & $\mathbf{P Q}(\mathbf{g})$ & Psat(g) & $\mathbf{P i}(\mathbf{g})$ \\
\hline \multicolumn{7}{c}{ Conformação sem a utilização de vácuo. } \\
\hline 34 & 7,6470 & 3,2540 & 5,5380 & 164,6300 & 187,1500 & 98,1000 \\
\hline 35 & 7,7120 & 3,2730 & 5,4630 & 154,9200 & 180,2500 & 90,3100 \\
\hline 36 & 7,5690 & 3,2780 & 5,4990 & 158,5400 & 179,0100 & 91,3400 \\
\hline Média & $\mathbf{7 , 6 4 2 7}$ & $\mathbf{3 , 2 6 8 3}$ & $\mathbf{5 , 5 0 0 0}$ & $\mathbf{1 5 9 , 3 6 3 3}$ & $\mathbf{1 8 2 , 1 3 6 7}$ & $\mathbf{9 3 , 2 5 0 0}$ \\
\hline \multicolumn{7}{c}{ Conformação com a utilização de vácuo. } \\
\hline 10 & 7,8680 & 3,2580 & 5,4820 & 171,1200 & 192,3800 & 103,3300 \\
\hline 11 & 7,7340 & 3,2470 & 5,3890 & 175,7700 & 197,3700 & 106,8200 \\
\hline 12 & 7,7940 & 3,2580 & 5,4020 & 179,6100 & 201,9700 & 107,7000 \\
\hline 13 & 8,1090 & 3,2760 & 5,4010 & 174,1300 & 195,9900 & 105,0900 \\
\hline Média & $\mathbf{7 , 8 7 6 3}$ & $\mathbf{3 , 2 5 9 8}$ & $\mathbf{5 , 4 1 8 5}$ & $\mathbf{1 7 5 , 1 5 7 5}$ & $\mathbf{1 9 6 , 9 2 7 5}$ & $\mathbf{1 0 5 , 7 3 5 0}$ \\
\hline
\end{tabular}

Tabela 2: Dimensões, peso após queima, peso saturado e peso imerso dos blocos conformados sem vácuo, com vácuo e queimados a $1000^{\circ} \mathrm{C}$.

\begin{tabular}{ccccccc}
\hline $\begin{array}{c}\text { Corpo } \\
\text { de } \\
\text { prova }\end{array}$ & $\begin{array}{c}\text { Comprimento } \\
(\mathbf{c m})\end{array}$ & $\begin{array}{c}\text { Largura } \\
(\mathbf{c m})\end{array}$ & $\begin{array}{c}\text { Altura } \\
\mathbf{( c m )}\end{array}$ & $\mathbf{P Q} \mathbf{( g )}$ & Psat(g) & Pi (g) \\
\hline \multicolumn{7}{c}{ Conformação sem a utilização de vácuo. } \\
\hline 37 & 7,4600 & 3,2380 & 5,3470 & 158,0000 & 170,9800 & 91,6300 \\
\hline 38 & 7,5820 & 3,2030 & 5,4720 & 159,4500 & 173,3000 & 92,3200 \\
\hline 39 & 7,3160 & 3,2220 & 5,3640 & 157,6800 & 169,5000 & 92,2000 \\
\hline Média & $\mathbf{7 , 4 5 2 7}$ & $\mathbf{3 , 2 2 1 0}$ & $\mathbf{5 , 3 9 4 3}$ & $\mathbf{1 5 8 , 3 7 6 7}$ & $\mathbf{1 7 1 , 2 6 0 0}$ & $\mathbf{9 2 , 0 5 0 0}$ \\
\hline \multicolumn{7}{c}{ Conformação com a utilização de vácuo. } \\
\hline 06 & 7,6430 & 3,1460 & 5,3900 & 164,2100 & 177,3800 & 87,0600 \\
\hline 09 & 7,6270 & 3,2250 & 5,3100 & 172,1000 & 184,9200 & 97,9500 \\
\hline 15 & 7,5850 & 3,2220 & 5,4300 & 172,6300 & 186,5000 & 96,4500 \\
\hline 17 & 7,4280 & 3,1960 & 5,4830 & 177,9800 & 191,2600 & 94,7100 \\
\hline Média & $\mathbf{7 , 5 7 0 8}$ & $\mathbf{3 , 1 9 7 3}$ & $\mathbf{5 , 4 0 3 3}$ & $\mathbf{1 7 1 , 7 3 0 0}$ & $\mathbf{1 8 5 , 0 1 5 0}$ & $\mathbf{9 4 , 0 4 2 5}$ \\
\hline \multicolumn{7}{c}{}
\end{tabular}


Tabela 3: Dimensões, peso após queima, peso saturado e peso imerso dos blocos submetidos à secagem rápida (foram conformados com vácuo e queimados a $800^{\circ} \mathrm{C}$ ).

\begin{tabular}{ccccccc}
\hline $\begin{array}{c}\text { Corpo } \\
\text { de } \\
\text { prova }\end{array}$ & $\begin{array}{c}\text { Comprimento } \\
\mathbf{( c m )}\end{array}$ & $\begin{array}{c}\text { Largura } \\
\mathbf{( c m )}\end{array}$ & $\begin{array}{c}\text { Altura } \\
\mathbf{( c m )}\end{array}$ & $\mathbf{P Q}(\mathbf{g})$ & Psat(g) & Pi (g) \\
\hline $1 S R$ & 7,5600 & 3,3940 & 5,6530 & 169,8500 & 190,1000 & 98,8300 \\
\hline $2 S R$ & 7,8150 & 3,2880 & 5,5220 & 169,8500 & 188,0400 & 97,5700 \\
\hline Média & $\mathbf{7 , 6 8 7 5}$ & $\mathbf{3 , 3 4 1 0}$ & $\mathbf{5 , 5 8 7 5}$ & $\mathbf{1 6 9 , 8 5 0 0}$ & $\mathbf{1 8 9 , 0 7 0 0}$ & $\mathbf{9 8 , 2 0 0 0}$ \\
\hline
\end{tabular}

Os resultados de absorção de água, porosidade aparente, massa específica aparente (MEA) e resistência à compressão dos corpos cerâmicos encontram-se nas Tabelas 4-6.

Tabela 4: Absorção de água, porosidade aparente, MEA e resistência à compressão dos blocos conformados sem vácuo, com vácuo e queimados a $800^{\circ} \mathrm{C}$.

\begin{tabular}{|c|c|c|c|c|c|}
\hline \multirow{2}{*}{$\begin{array}{l}\text { Corpo } \\
\text { de prova }\end{array}$} & \multirow{2}{*}{$\begin{array}{c}\text { Absorção } \\
\text { de água } \\
(\%)\end{array}$} & \multirow{2}{*}{$\begin{array}{l}\text { Porosidade } \\
\text { aparente(\%) }\end{array}$} & \multirow{2}{*}{ MEA (\%) } & \multicolumn{2}{|c|}{$\begin{array}{c}\text { Resistencia à } \\
\text { compressão (MPa) }\end{array}$} \\
\hline & & & & $\begin{array}{c}0,05 \\
\mathrm{MPa} / \mathrm{s}\end{array}$ & $5 \mathrm{MPa} / \mathrm{s}$ \\
\hline \multicolumn{6}{|c|}{ Conformação sem a utilização de vácuo. } \\
\hline 34 & 13,6792 & 25,2892 & 1,8487 & 0,40 & --- \\
\hline 35 & 16,3504 & 28,1632 & 1,7225 & 0,66 & $\overline{---}$ \\
\hline 36 & 12,9116 & 23,3489 & 1,8084 & 0,18 & --- \\
\hline Média & 14,31 & 25,60 & 1,79 & 0,41 & --- \\
\hline $\begin{array}{c}\text { Desvio } \\
\text { P. }\end{array}$ & 0,5428 & 1,3720 & 0,0285 & 0,24 & --- \\
\hline \multicolumn{6}{|c|}{ Conformação com a utilização de vácuo. } \\
\hline 10 & 12,4240 & 23,8742 & 1,9216 & 1,17 & --- \\
\hline 11 & 12,2888 & 23,8542 & 1,9411 & 1,13 & --- \\
\hline 12 & 12,4492 & 23,7191 & 1,9053 & --- & 1,18 \\
\hline 13 & 12,5538 & 24,0484 & 1,9156 & --- & 1,48 \\
\hline Média & 12,4290 & 23,8740 & 1,9209 & 1,15 & 1,33 \\
\hline $\begin{array}{c}\text { Desvio } \\
\text { P. }\end{array}$ & 0,0918 & 0,1232 & 0,0042 & 0,028 & 0,212 \\
\hline
\end{tabular}

Conforme observado na Tabela 4 , os valores médios obtidos de porosidade aparente (PA) são maiores nos corpos de prova extrudados sem vácuo do que nos extrudados com vácuo. O que já se esperava, pois no processo de extrusão sem vácuo a quantidade de ar retida no interior da massa, durante a conformação, é maior do que na extrusão com vácuo.

Como porosidade e absorção de água estão diretamente relacionadas, uma vez que os poros abertos são responsáveis pela absorção de água, os corpos de prova 
extrudados sem vácuo apresentaram também uma maior absorção de água do que os extrudados com vácuo.

Tabela 5: Absorção de água, porosidade aparente, MEA e resistência à compressão dos blocos conformados sem vácuo, com vácuo e queimados a $1000^{\circ} \mathrm{C}$.

\begin{tabular}{|c|c|c|c|c|c|}
\hline \multirow{2}{*}{$\begin{array}{l}\text { Corpo } \\
\text { de prova }\end{array}$} & \multirow{2}{*}{$\begin{array}{c}\text { Absorção } \\
\text { de água } \\
\text { (\%) }\end{array}$} & \multirow{2}{*}{$\begin{array}{l}\text { Porosidade } \\
\text { aparente } \\
\text { (\%) }\end{array}$} & \multirow{2}{*}{ MEA (\%) } & \multicolumn{2}{|c|}{$\begin{array}{c}\text { Resistencia à } \\
\text { compressão (MPa) }\end{array}$} \\
\hline & & & & $\begin{array}{c}0,05 \\
\mathrm{MPa} / \mathrm{s}\end{array}$ & $5 \mathrm{MPa} / \mathrm{s}$ \\
\hline \multicolumn{6}{|c|}{ Conformação sem a utilização de vácuo. } \\
\hline 37 & 8,2152 & 16,3579 & 1,9912 & 0,82 & --- \\
\hline 38 & 8,6861 & 17,1030 & 1,9690 & 0,84 & --- \\
\hline 39 & 7,4962 & 15,2911 & 2,0398 & 0,22 & --- \\
\hline Média & 6,1223 & 12,2188 & 1,5011 & 0,63 & --- \\
\hline $\begin{array}{l}\text { Desvio } \\
\text { P. }\end{array}$ & 0,5084 & 0,7544 & 0,0344 & 0,35 & --- \\
\hline \multicolumn{6}{|c|}{ Conformação com a utilização de vácuo. } \\
\hline 06 & 8,0202 & 14,5815 & 1,8181 & 1,84 & --- \\
\hline 09 & 7,4492 & 14,7407 & 1,9788 & 1,76 & --- \\
\hline 15 & 8,0345 & 15,4026 & 1,9170 & --- & 2,09 \\
\hline 17 & 7,4615 & 13,7545 & 1,8434 & --- & 2,39 \\
\hline Média & 7,7414 & 14,6198 & 1,8893 & 1,80 & 2,24 \\
\hline $\begin{array}{l}\text { Desvio } \\
\text { P. }\end{array}$ & 0,3951 & 0,5847 & 0,0179 & 0,056 & 0,212 \\
\hline
\end{tabular}

Comparando os valores de porosidade, absorção de água e resistência à compressão entre os blocos queimados a $800^{\circ} \mathrm{C}$ e $1000^{\circ} \mathrm{C}$, respectivamente, observou- se que os blocos queimados a $1000^{\circ} \mathrm{C}$ possuem menor porosidade, caracterizando um material mais denso, consequentemente com menor absorção de água e maior resistência mecânica; podendo estes resultados estar relacionados com o fato de que na temperatura mais elevada, há maior probabilidade de gerar fase líquida, que por sua vez facilita e acelera a difusão atômica permitindo a ocorrência de transformações de fases e, consequentemente, a obtenção de uma microestrutura mais densa e resistente. 
Tabela 6: Absorção de água, porosidade aparente, MEA e resistência à compressão dos blocos submetidos à secagem rápida (foram conformados com vácuo e queimados a $800^{\circ} \mathrm{C}$ ).

\begin{tabular}{|c|c|c|c|c|c|}
\hline \multirow{2}{*}{$\begin{array}{l}\text { Corpo } \\
\text { de prova }\end{array}$} & \multirow{2}{*}{$\begin{array}{c}\text { Absorção } \\
\text { de água } \\
(\%)\end{array}$} & \multirow{2}{*}{$\begin{array}{c}\text { Porosidade } \\
\text { aparente } \\
(\%)\end{array}$} & \multirow{2}{*}{ MEA (\%) } & \multicolumn{2}{|c|}{$\begin{array}{c}\text { Resistencia à } \\
\text { compressão (MPa) }\end{array}$} \\
\hline & & & & $\begin{array}{c}0,05 \\
\mathrm{MPa} / \mathrm{s}\end{array}$ & $5 \mathrm{MPa} / \mathrm{s}$ \\
\hline 1SR & 11,9223 & 22,1869 & 1,8610 & 1,10 & --- \\
\hline $2 S R$ & 10,7094 & 20,1061 & 1,8774 & --- & 2,10 \\
\hline Média & 11,3159 & 21,1465 & 1,8692 & 1,10 & 2,10 \\
\hline $\begin{array}{l}\text { Desvio } \\
\text { P. }\end{array}$ & 0,8576 & 1,4714 & 0,0116 & --- & --- \\
\hline
\end{tabular}

De acordo com os valores de resistência apresentado na Tabela 4 (blocos extrudados com vácuo) e na Tabela 6 , observa-se que o tempo de secagem teve influência direta na resistência mecânica dos corpos cerâmicos, uma vez que os valores de resistência obtidos nos corpos submetidos à secagem rápida foram inferiores aos submetidos à secagem lenta. Este fato pode estar associado ao tempo insuficiente para eliminação das águas livres ou de conformação, que são responsáveis pela retração das peças. O tempo insuficiente de secagem podem provocar tensões no material, quando submetido à queima, favorecendo 0 aparecimento de trincas, e consequentemente, reduzindo a resistência do material.

Ao analisar os valores de resistência apresentados na Tabela 4-6, observa-se que os blocos submetidos a uma taxa de aplicação de força de $5 \mathrm{Mpa} / \mathrm{s}$ obtiveram valores de resistência a compressão superiores aos blocos submetidos à uma taxa de $0,05 \mathrm{Mpa} / \mathrm{s}$. Isto pode ser explicado pelo fato de que, em taxas muito elevadas à probabilidade de encontrar os defeitos presentes no material (pequenos defeitos) diminui e, em consequência disto, mascaram a resistência real do material.

\section{CONCLUSÕES}

Com base nos resultados obtidos, após realização dos ensaios para a determinação da absorção de água, porosidade aparente, massa específica aparente, resistência à compressão e retração linear. Pode-se concluir que:

- A utilização de câmara de vácuo melhora a resistência do material. Visto que, os resultados de porosidade aparente e absorção de água foram maiores nos corpos de prova extrudados sem vácuo do que nos extrudados com vácuo.

- A temperatura de queima a que o material é submetido influência nas propriedades do mesmo. Os blocos queimados a $1000^{\circ} \mathrm{C}$ apresentaram maior resistência à compressão e menor porosidade, caracterizando um material mais denso e consequentemente, com menor absorção de água.

- O tempo de secagem afeta a resistência mecânica dos blocos cerâmicos. Tempo insuficiente de secagem podem provocar tensões no material na etapa de queima, favorecendo 0 aparecimento de trincas, e consequentemente, reduzindo a resistência do material. 
- A taxa de aplicação de força influência nos resultados de resistência obtidos. Isto pode ser explicado pelo fato de que, em taxas muito elevadas à probabilidade de encontrar os defeitos presentes no material (pequenos defeitos) diminui e, em consequência disto, mascaram a resistência real do material.

\section{Agradecimentos}

A CAPES pelo incentivo financeiro.

\section{REFERÊNCIAS}

1 RIBEIRO, M.J.; FERREIRA, A.A.L.; LABRINCHA, J.A. Aspectos Fundamentais sobre a Extrusão de Massas de Cerâmicas Vermelhas. Cerâmica Industrial, v. 8, $\mathrm{n}^{\circ}$ 1, pg. 37-42, Janeiro/Fevereiro, 2003.

2 SANTOS, R, C. Estudo Comparativo de Blocos Cerâmicos para Alvenaria Produzidos na Paraíba e no Rio Grande do Norte. 2010. 71 f.. Relatório (Estágio Integrado) - Universidade Federal de Campina Grande, Campina Grande, 2010.

3 WIECK, R.; DUAILIBI FH,J. Extrusão em Cerâmica Vermelha: Princípios Básicos, Problemas e Soluções. Cerâmica Industrial, v. 18, n³, pg. 16-23, Maio/Junho, 2013.

4 FONSECA, J. F. et al. Manual para a Produção de Cerâmica Vermelha. Florianópolis, Universidade Federal de Santa Catarina/ SEBRAE-SC, 1994,81p.

5 VIEIRA, C. M. F. et al. Avaliação da Secagem de Cerâmica Vermelha através da Curva de Bigot. Cerâmica Industrial, v. 8, n², pg. 42-46, Janeiro/Fevereiro, 2003.

6 ROMAN, H. R. Determinação das Características Físicas e Análise Estatística da Capacidade Resistente de Tijolos Cerâmicos Maciços. 1983.102 f.. Dissertação (Mestrado) - Universidade Federal do Rio Grande do Sul, Porto Alegre, 1983.

7 VAN VLACK, L. H. Propriedades dos Materiais Cerâmicos. Tradução de Cid Silveira e Shiroyuki Oniki. São Paulo: Edgard Blucher, 1973.

8 ASSOCIAÇÃO BRASILEIRA DE NORMAS TÉCNICAS. NBR 15270-3:

Componentes cerâmicos. Parte 3: Blocos cerâmicos para alvenaria estrutural e de vedação - Métodos de ensaio. Rio de Janeiro, 2005. 